\title{
A Confirmatory Measurement Technique
for Highly Enriched Uranium
}

J. K. Sprinkle, Jr.

\section{DISCLAIMER}

\begin{abstract}
This report was prepared as an account of work sponsored by an agency of the United States Government. Neither the United States Government nor any agency thereof, nor any of their employees, makes any warranty, express or implied, or assumes any legal liability or responsibility for the accuracy, completeness, or usefulness of any information, apparatus, product, or process disclosed, or represents that its use would not infringe privately owned rights. Reference herein to any specific commercial product, process, or service by trade name, trademark, manufacturer, or otherwise does not necessarily constitute or imply its endorsement, recommendation, or favoring by the United States Government or any agency thereof. The views and opinions of authors expressed herein do not necessarily state or reflect those of the United States Government or any agency thereor.
\end{abstract}




\section{CONTENTS}

ABSTRACT . . . . . . . . . . . . . . . . 1

I. INTRODUCTION . . . . . . . . . . . . . . . . 1

II. MEASUREMENT REQUIREMENTS AND MEUTRON-BASED

TECHNIQUES . . . . . . . . . . . . . . 3

III. THE THREE-GAMMA TECHNIQUE . . . . . . . . . . 5

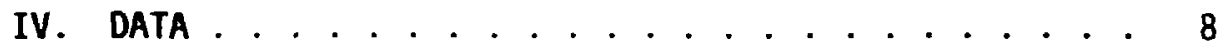

v. CONCLUSIONS .................. 12

VI. FUTURE PLANS . . . . . . . . . . . . . . 12

ACKNONLEDGEMENTS . . . . . . . . . . . . . 13

REFEREMCE ................. 13 


\title{
A CONFIRMATORY MEASUREMENT TECHNIQUE FOR HIGHLY ENRICHED URANIUM
}

\author{
by
}

J. K. Sprinkle, Jr.

\begin{abstract}
This report descrives a confirmatory measurement technique for measuring uranium items in their shipping containers. The measurement consists of a weight verification and the detection of three gamma rays. The weight can be determined very precisely, thus it severely constrains the options of the diverter who might want to imitate the gamma signal with a bogus item. The $185.7-\mathrm{keV}$ gamma ray originates from $235 \mathrm{U}$, the $1001 \mathrm{keV}$ originates from a daughter of $238 \mathrm{~J}$, and the $2614 \mathrm{keV}$ originates from a daughter of $232 \mathrm{U}$. These three gamma rays exhibit widely different attenuation properties, they correlate with enrichment and total uranium mass, and they rigorously discriminate against a likely diversion scenario (low-enriched uranium substitution). These four measured quantities, when combined, provide a signature that is very difficult to counterfeit.
\end{abstract}

\section{INTRODUCTION}

Confirmatory measurements are measurements that have less than state-ofthe-art accuracy. They are used in situations in which the benefits of attaining the best accuracy do not justify the associated expense. The ability to obtain a result quickly and nonintrusively. is judged to be more important than achieving the best accuracy. The most accurate confirmatory measurements provide results that are better than $1 \%$, while at the other extreme some confirmatory measurements yield order-of-magnitude results. This wide range of measurement accuracies is acceptable because different situations require different tradeoffs between the costs of the results and the quality of the results. The more accurate measurements are more expensive in terms of both time and money. 
Routine use of measurement results often requires that results from different instruments be compared. However, sometimes it can be more informative to compare less accurate results from identical instruments. In this type of comparison, the user can take advantage of measurement precisions, which are much smaller than the measurement accuracies. This can be a very rigorous comparison, in spite of relatively poor measurement accuracy, if the two measurements being compared are susceptible to the same extent to the same biases.

Two measurement situations at $\mathrm{Y}-12$ are the focus of this confirmatory instrument design. Shipper/receiver transactions and internal inventory confirmations are the primary measurement objectives. However, there has been an attempt to not preclude any type of uranium confirmatory measurement. This technique should also perform well for low-enriched uranium (LEU) and for uranium-bearing materials that are not quite as pure as those typically found at $Y-12$.

A confirmatory measurement for highly enriched uranium (HEU) will provide an excellent supplement to the current shipper/receiver (S/R) measurements. The intent of the present regulations regarding material transfers between facilities is that a receiver perform input accountability measurements within 10 days of receipt in order to detect potential shipper/receiver differences in a timely fashion. When the entire safeguards picture is included in the analysis, this requirement is not always the most desirable thing to do.l In fact, it is not always a possible thing to do. An alternative procedure, based on confirmatory measurements, will help to clarify the issues if a S/R difference occurs. When the shipper's value is compared to the receiver's value, are the two measurements of the same item or of different items? If a S/R difference occurs, the most important issue that needs to be resolved is whether the uifference is due to a bias between the two measurements or is due to material's being diverted. A successful confirmatory measurement would eliminate the possibility that material was diverted.

A confirmatory measurement for HEU can also be used to verify that an inventory item has not been tampered with. Periodic inventories currently rely more heavily on physical security, such as seals and proteciive barriers, instead of on measurements of the special nuclear material (SNM). A rapid semi-quantitative measurement of the SNM will significantly increase the confidence that the item still contains the original material. In addition to the inventory verification, it should also be possible to improve the knowledge of 
some older items, which have a poorly known quantity of SNM. Sometimes it is not cost effective to attempt a careful analytica) determination (in fact it is often not possible to use standard analytical techniques), but a confirmatory measurement could provide a measured value where none at present exists.

\section{MEASUREMENT REQUIREMENTS AND NEUTRON-BASED TECHNIQUES}

In investigating candidate techniques for confirmatory measurements of HEU at $Y-12$, the following constraints seem to be reasonable based on their cost and capabilities. The technique must be less expensive and faster than traditional input accountability measurements. In order for the confirmatory technique to be capable of distinguishing diversions, good measurement precision must be retained. However, the measurement accuracy requirements are not as clear. At a minimum, the measurement needs to unconditionally verify the presence of HEU. In addition, a semi-quantitative determination of the amount of HEU gives added assurance that the item has not been tampered with. However, it lis already been decided that a very accurate determination of the amount of HEU (the receiver's input accountability measurement) has an excessive cost for the benefits it delivers. For nondestructive assay (NDA) measurements in general, $1 \%$ is a reasonable precision; for these verification measurements, $10 \%$ (or better) hias been chosen as the accuracy goal. For $S / R$ transfers, the input accountability result will be determined by traditional means when it is required by the process. This approach verifies that nothing was altered during transport, and it does not compromise the input accountability values. Consequently, it provides a reasonable test for potential S/R differences. For internal inventory verifications, this approach provides a measurement where none at present exists.

The quantities of special nuclear material in a typical shipping container are large. Initially, this fact led to a consideration of neutron-based techniques since neutrons generally penetrate large dense objects better than gamma rays. The passive neutron signal from uranium is quite weak; as a consequence, most neutron-based assays of uranium require active interrogation. An assessment of the potential active neutron techniques disclosed that none of the already built instruments were satisfactory for this measurement problem. Instruments that achieved tine stated accuracy goal of $10 \%$ with an instrument 
based on a $4 \pi$ geometry were either in the $\$ 400 k$ (shuffler) to $\$ 1 M$ (differential die-away technique) cost range or required that the shipping containers be opened and the items be measured in the significantly smaller inner container [active well coincidence counter (AWCC) at $\$ 150 \mathrm{k}$ ]. The latter choice was quite objectionable for several reasons. First, the increased handing (additional manpower) required to open the shipping containers and extra time just for a verification measurement increases the opportunities of diversion, not to mention the opportunities for contamination. In addition, the breaking of the shipper's seal with no subsequent accountability measurement causes severe consternation among those who have seen an occasional disagreement in the past over shipper's compared to receiver's values. Finally, several items are too large to fit into the wells of present AWCCS. This would make the latter option somewhat more difficult than purchasing a commercial instrument. On the other hand, the former choice seemed too expensive for the DOE/NRC complex to support (one or more instruments per uranium facility). It also appears to provide an instrument that may provide better accuracy than the problem warrants. It might well be simpler and more cost effective to increase the traditional input accountability capability to the level required to meet the 10-day rule instead of choosing a sophisticated neutron technique. of all the active neutron techniques, only one merits further investigation in the context of HEU, confirmatory measurements. A detector based on a plastic scintillator could possibiy achieve adequate counting statistics. However, further investigation is needed to estimate count rates and some technical problems related to stability need to be satisfactorily resolved before a conceptual design of an instrument can be made. This investigation will proceed in parallel with the proposed instrument.*

A consideration of potential gamma-ray-based techniques suggested this was a superior alternative to the neutron-based techniques in light of two features of this measurement problem. First, most of the samples under consideration are large. Kilogram quantities of HEU emit significant quantities of the higher-energy gamma rays even though the branching ratios are low. Second, the standard shipping containers span a very narrow range of geometries. Almost

\footnotetext{
*This investigation has shown that electronic stability is still a major problem and that in order to achieve reasonable counting statistics, a large expensive unit is required.
} 
all shipping containers for HEU are limited by criticality safety constraints to a 15-cm-diam pipe centered in some type of barrel. The SNM must be in the pipe and a low-density packing material fills the barrel outside the pipe. The quality of construction is such that the pipe is very close to the actual center of the barrel, and the barrels generally are removed from service if they sustain even minor damage. This geometrical constraint limits the magnitude of the variation of the self-attenuation in these measurements significantly.

If the $S / R$ issue were the only consideration, the accuracy requirement stated above could be relaxed, which would result in a minor loss in the safeguards effectiveness. It would then be reasonable to choose simpler, portable instruments such as an active neutron coincidence collar or a small multichannel analyzer (MCA) with a single $\mathrm{NaI}(\mathrm{TI})$ detector. The efficacy of the measurements would rely on the repeatability of the raw instrument values rather than the SNM determination. However, the second desired use for this instrument (inventory confirmation) requires that the accuracy requirement be retained or even tightened. Preliminary data indicate that this proposed design can achieve a measurement accuracy of $10 \%$ or better. Further investigation with a prototype instrument will evaluate how much improvement in the measurement accuracy can be achieved.

\section{THE THREE-GAMMA TECHNIQUE}

The candidate measurement technique consists of a weight measurement and the detection of three gamma rays of different energies. The items will be measured in the shipping containers; consequently, the tamper-indicating seal already in use will be undisturbed. The three gamma rays are emitted by all enriched uranium. The $185.7-\mathrm{keV}$ gamma ray originates from $235 \mathrm{U}$ decay, the $1001 \mathrm{keV}$ originates from a daughter of $238 \mathrm{U}$, and the 2614 originates from a daughter of $232 \mathrm{U}$. The origin of the $232 \mathrm{U}$ in the HEU is from reactor returns. Consequently, the intensity of the 2614-keV gamma ray is not easily predicted. Therefore, it will be used as a spike in this technique. The three gamma rays exhibit widely different attenuation properties. Table I lists the thicknesses of uranium metal and powdered oxide that will attenuate the three gamma-ray intensities by a factor of 500 . Thicknesses that exceed these values contain material that does not contribute to the measured signal. 
It is clear that a different fraction of very large samples contributes to the measured response of each gamma ray. However, it is helpful to remember the geometrical constraints provided by the standard shipping containers. A 15-cm-diam pipe centered in a 113- or 208-l barrel, with the SNM in the pipe and a low-density matrix outside the pipe, is less than the penetrability of the two higher-energy gamma rays in all materials except pure uranium metal.

The preliminary data taken with a single detector and a portable MCA indicate that the $10 \%$ accuracy goal should be achievable with a more sophisticated instrument. In orde; to improve the measurement accuracy, the prototype instrument will be more complex. Figure 1 is a conceptual design of the instrument. The three different sizes of shipping containers are shown on a simple drum rotator. The 113- $\ell$ drum is represented by the dashed lines and the 208- $\ell$ and double 208-l (416-l) drums are represented by the solid outlines. The four detectors will be spaced vertically so that the instrument will have a flat response in the vertical dimension. The barrel being measured will be rotated to average over radial variations. The instrument will be controlled by a minicomputer. This will allow for user-friendly automated operation and a more complicated data reduction algorithm if it is necessary.

The expected measurement scenario assumes that these instruments are adequately shielded from background sources. Since they will be used in close proximity to many other containers and the 2614-keV gamma ray is highly penetrating, a large shield will need to be censtructed. Current implementation designs are based on a movable 61-cm-thick concréte shielding wall in addition to $10 \mathrm{~cm}$ of lead surrounding each detector. The four-detector assembly will weigh approximately $2273 \mathrm{~kg}$.

TABLE I

INFINITE THICKNESS OF URANIUM FOR DIFFERENT GAMMA ENERGIES

\begin{tabular}{|c|c|c|}
\hline Gamma & & ess \\
\hline $\begin{array}{r}\text { energy } \\
(\mathrm{keV})\end{array}$ & $\begin{array}{l}\text { metal } \\
(\mathrm{cm})\end{array}$ & $\begin{array}{l}\text { oxide } \\
(\mathrm{cm})\end{array}$ \\
\hline $\begin{array}{r}186 \\
1001 \\
2614\end{array}$ & $\begin{array}{l}0.2 \\
4.2 \\
7.2\end{array}$ & \\
\hline
\end{tabular}




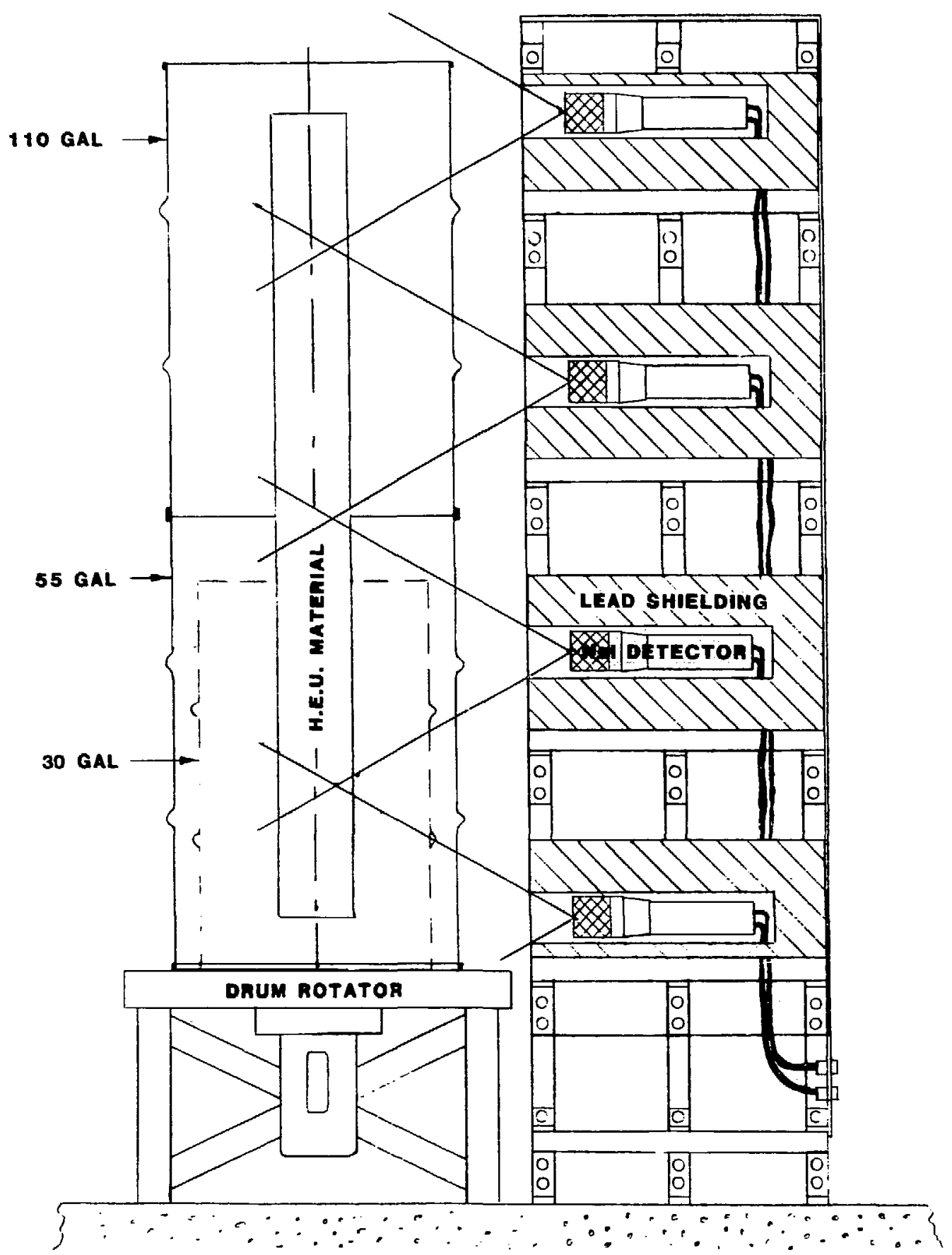

\section{H.E.U. CONFIRMATORY INSTRUMENT DE İECTORS}

Fig. 1. A conceptual design for the confirmatory instrument for uranium shipments. Four 7.5 by $7.5-\mathrm{cm} \mathrm{NaI}(\mathrm{TI})$ detectors are arranged in a yertical stack to provide a uniform detection response over the height of the shipping container. Each detector is collimated and shielded with 10-cm-thick lead. 
It is not clear at present that the shipper and the receiver must use identical instruments. If they do, the shipper will pack the material in the shipping container, seal the container, and perform the verification with his instrument. The containers and the verification data will be sent separately to the receiver, who will perforin the measurements with his instrument and compare the two sets of results within 10 days. If they do not use identical instruments, the accuracy requirements of the receiver's confirmatory measure ment will be stricter since the comparison will be in units of grams instead of counts per second. These requirements are not yet defined.

\section{DATA}

The three gamma rays have been measured in HEU at Los Alamos, at Pantex, and at $Y-12$ in order to evaluate the feasibility of this proposed technique. Typically, the data were obtained with a low-resolution gamma-ray detection system connected to portable electronics. The majority of the data were acquired with 7.5 by $7.5-\mathrm{Cm} N a I(T 1)$ detectors connected to a portable MCA. The sample-to-detector geometry varied, but typically the detectors were $30 \mathrm{~cm}$ above the bottom of the shipping drum and 5 to $41 \mathrm{~cm}$ away from the shipping drum. In some cases the front of the detector had a filter of $0.03-\mathrm{cm}$ copper and $0.24-\mathrm{cm}$ cadmium. More filtering would have been useful to reduce the $185.7 \mathrm{keV}$ intensity. Typically, the detector was poorly collimated, but it had a lead shield approximately $2 \mathrm{~cm}$ thick. Most of the sample material was not freshly processed.

Typical counting rates are listed in Table II.

TABLE II

NOMINAL COUNTING RATES FOR TYPICAL SHIPPING CONTAINERS

$\begin{array}{cc}\begin{array}{c}\text { Gamma Energy } \\ (\mathrm{keV})\end{array} & \begin{array}{c}\text { Count Rate } \\ \text { (counts/s/kg) }\end{array} \\ 186 & 100(235 \mathrm{U}) \\ 1001 & 10(238 \mathrm{U} \\ 2614 & 5(232 \mathrm{U})\end{array}$


Figure 2 shows the 1001-keV response as a function of $238 \mathrm{U}$ mass in grams. The metal samples were inside 113-l shipping barrels. The different symbols correspond to different enrichments. This is the gamma-ray result that could be used to determine the sample mass. These data have an average deviation of $16 \%$ from a straight line fit. Figure 3 shows the $1001-\mathrm{keV}$ response for some UNH and oxide samples. The three symbols correspond to different enrichments. Some of the apparent inconsistencies shown by the data represented by the solid dots are due to measurement geometry effects.

The 186-keV gamma ray also correlates with sample mass, but in a more complex way. The measured 186-keV radition does na rirtnate from the cutire sample because of the severe self-attenuation in uranium. However, the purity of the samples and their geometrical uniformity combine to offset the attenuation effects in most cases. Figure 4 shows the $186-k e V$ response as a function of sample mass for three different measurement geometries. The nine data points for $6700 \mathrm{~g} 235 \mathrm{U}$ correspond to measurements on the same barrel in different radial directions.

Figure 5 shows the $2614-\mathrm{keV}$ response as a function of $235 \mathrm{u}$ mass. This illustrates that the inost penetrating gamma ray does not correlate with sample mass. But in conjunction with two gamma rays that do correlate with sample mass, it is an excellent tracer. A potential diverter has no way to predict what the 2614-keV count rate should be. Therefore, its count rate must be explicitly measured before one can know what value is needed in the bogus item.

Another result that can be obtained from an overview of the measurements on large samples, independent of the sample-to-detector geometry, is that the $185.7 / 1001$ count rate ratio provides a clear indication of the semple enrichment. Table III summarizes the results.

TABLE III

THE $185 / 1001$ COUNT RATE RATIO VS ENRICHMENT

\begin{tabular}{ccc}
$\begin{array}{c}\text { Enrichment } \\
(\%)\end{array}$ & & \multicolumn{2}{c}{$185 / 1001$ count rate ratio } \\
\cline { 2 - 3 } & minimum & maximum \\
$<50$ & 10 & 17 \\
$50-90$ & 40 & 96 \\
$>90$ & 152 & 1000
\end{tabular}




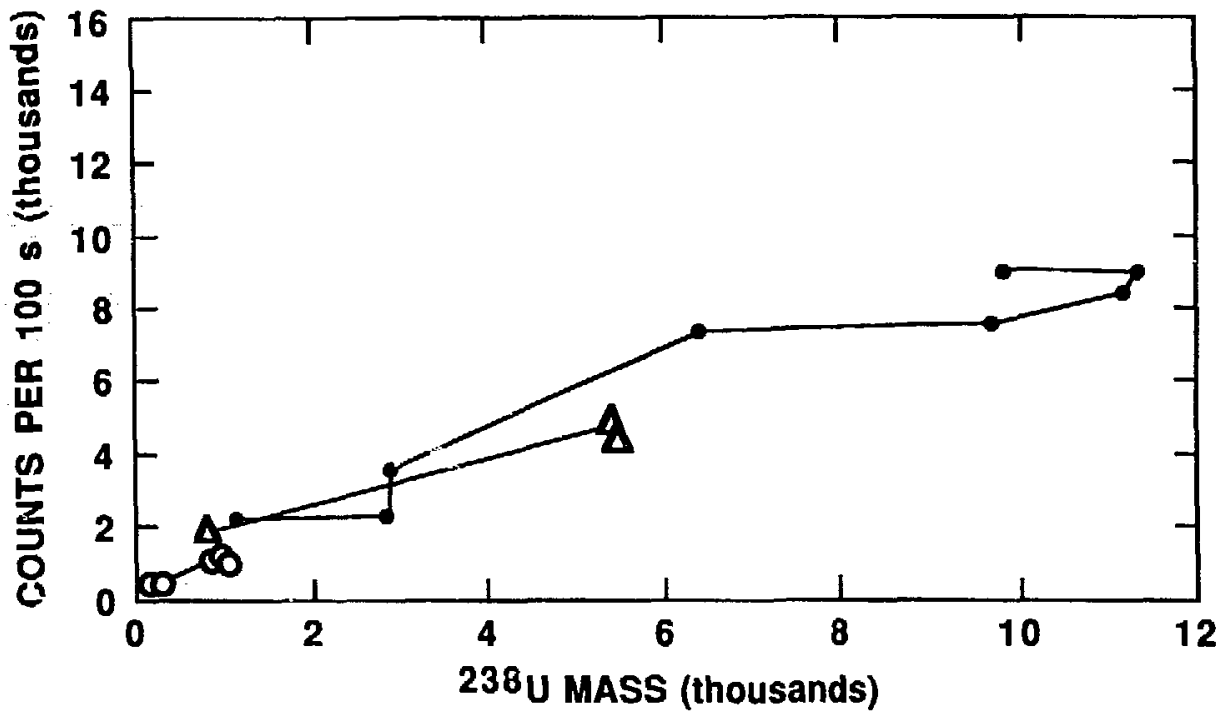

Fig. 2. The 1001-keV net counts in $100 \mathrm{~s}$ as a function of 238 $u$ mass in uranium metal. The different symbols correspond to different enrichments.

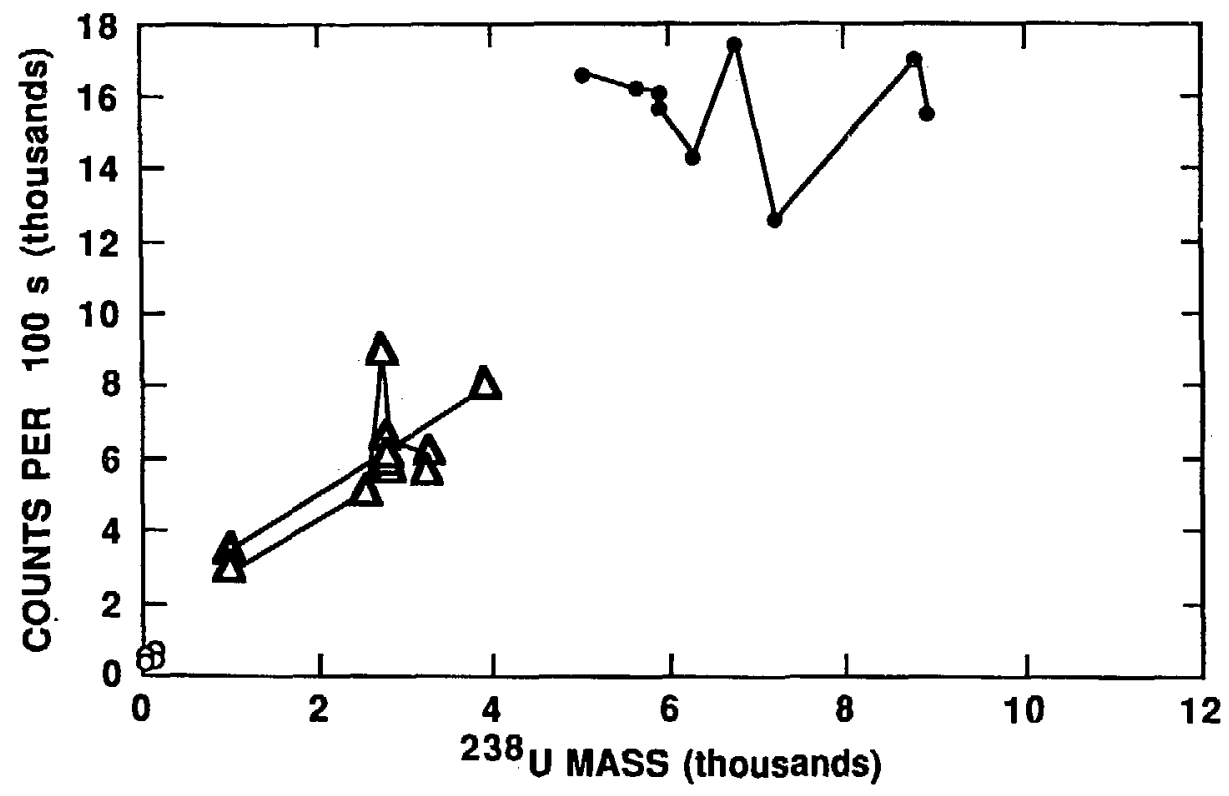

Fig. 3. The 1001-keV net counts in $100 \mathrm{~s}$ as a function of $238 \mathrm{U}$ mass in oxide and UNH. The three different symbols correspond to three different enrichments. The plus ( + ) signs correspond to several measurement geometries. 


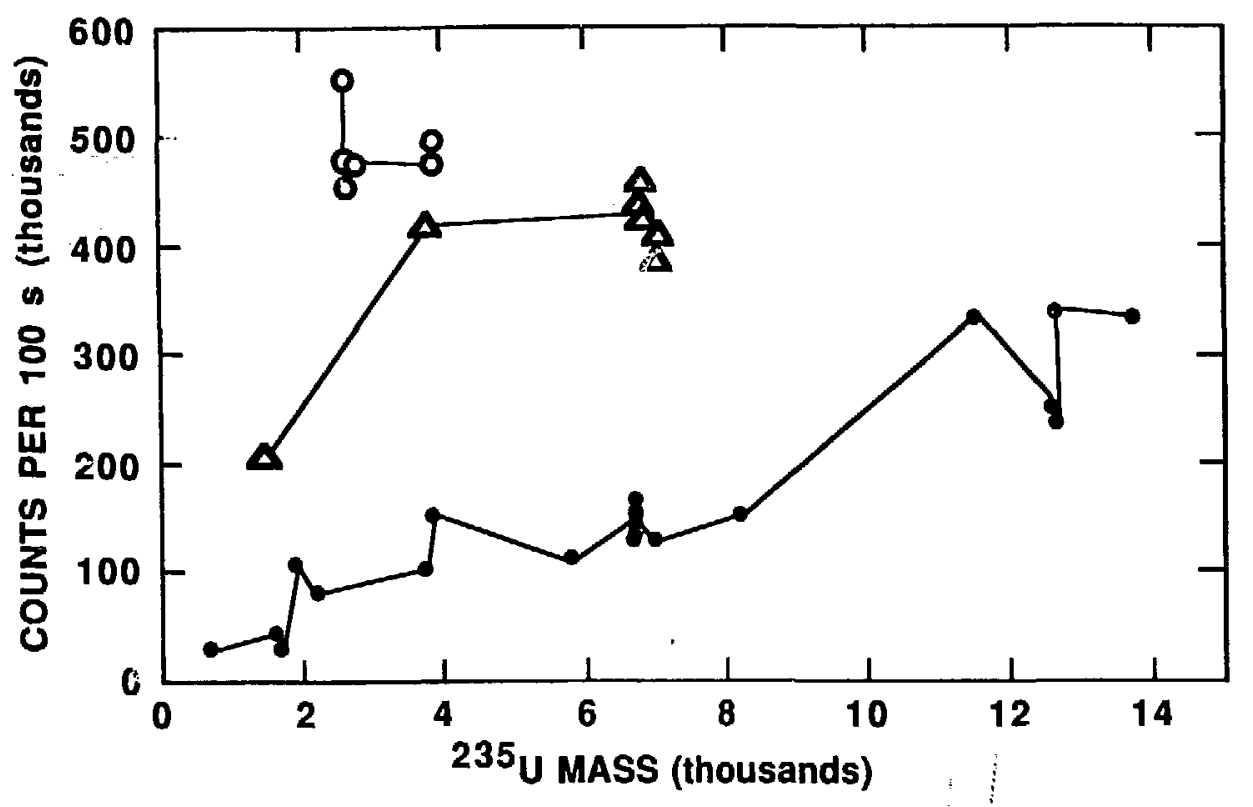

Fig. 4. The 185.7-keV net counts in $100 \mathrm{~s}$ as anction of $235 \mathrm{U}$ mass in uranium metal. The different symbols correspond to different measurement geometries.

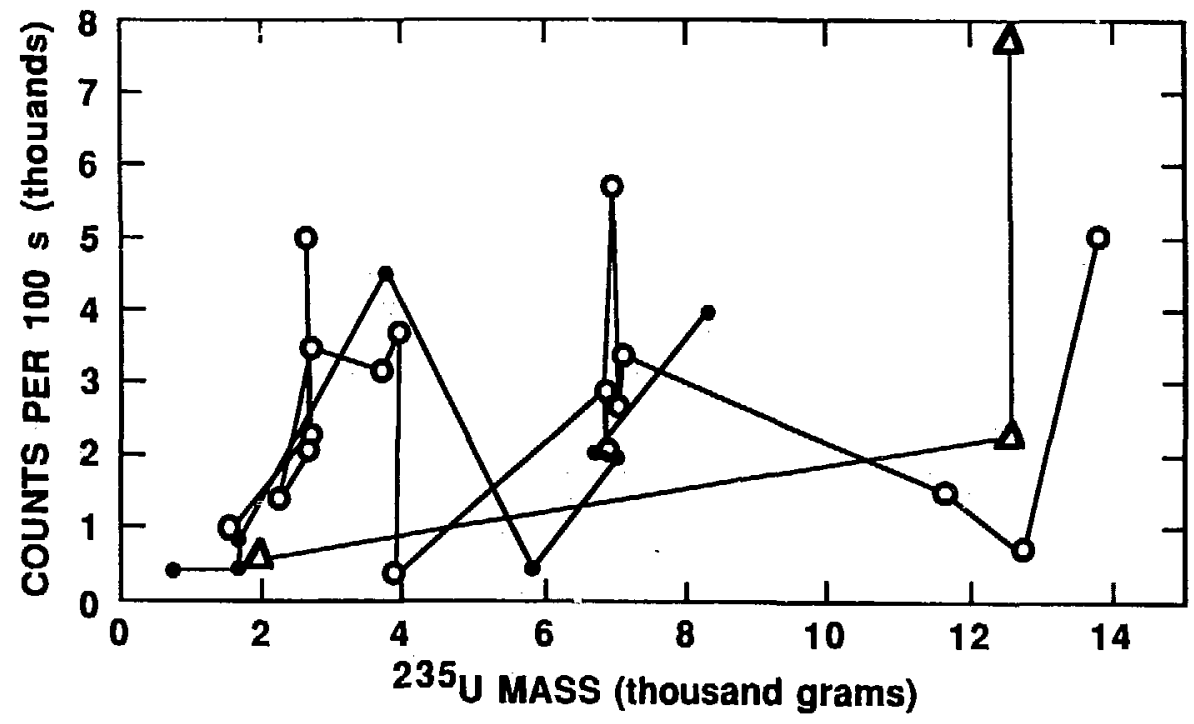

Fig. 5. The 2614-keV net counts in $100 \mathrm{~s}$ as a function of $235 \mathrm{U}$ mass in uranium metal. The different symbols correspond to different measurement geometries. 


\section{v. CONCLUSIONS}

Several features of these data warrant more careful study. The response per unit mass may depend on the chemical form or the enrichment of the sample. Either the $1001 \mathrm{keV}$ or the $2614 \mathrm{keV}$ may change with time depending on the status of the equilibrium between the uranium parent and tro daughter, which emits the gamma ray. The relevant half-lives are 24 days and 70 years, respectively. Nearly all of the $i$ tems at $Y-12$ and received by $Y-12$ are sufficiently old such that the 24-day half-life decay has reached equilibrium. In any event, the appropriate correction can be calculated if necessary. The signal from the gamma rays may change as the material settles during shipping and handling.

These data indicate that the issues requiring further study are primarily accuracy effects. This technique can achieve high precision and is difficult to fool with bogus items. The 1001-keV gamma ray has sufficient intensity to discriminate against the simple diversion scenario of LEU substitution. The expected accuracy of the weight measurement of 1 part in $10^{4}$ or beiter allows a $10-g$ uncertainty on a $100-\mathrm{kg}$ drum containing $1 \mathrm{~kg}$ of SNM. This represents a $i \%$ SNM weight verification on the item while it is in the container.

\section{FUTURE PLANS}

The: - is sufficient information at this point to justify building a prototype instrument to evaluate. The instrument that will implement this technique will rotate the barrel in front of a vertical row of detectors. It may be useful to combine the data from each detector or it may be better to compare the data from each detector separately. At this time it is not clear whether simple peak-area analysis techniques or response functions would be the preferred raw-data-reduction methoo. Projected count times are of the order of $200 \mathrm{~s}$. Clearly, massive shielding is required to measure the 2614-keV gamma ray in one container when there are many similar containers in the immediate vicinity: Initially, the evaluation will be concerned with the accuracy and precision limits of the instrument. Then calibration procedures, pass/ fail criteria, and settling effects of the SNM during handling will be evaluated. Eventually two prototypes will be needed, in order to evaluate results 
from actual shipments. The current candidates for evaluation of actual shipments are $Y-12$ and Rocky flats. At the end of the evaluation, we expect to understand the calibration procedures and the accuracy and precision capabilities of these instruments. Subsequently, a final design package will be produced for distribution.

\section{ACKNONLEDGMENTS}

The author would like to acknowledge the assistance of the NMC \& A Department at $Y-12$, in particular that provided by Steve Smith, Tom Brumfield, Gene Walker, Doug Watson, and Cynthia Gunn, and the support of his colleagues in the Safeguards Assay group at Los Alamos National Laboratory, in particular that provided by Phyllis Russo and Len Stovall.

\section{REFERENCE}

1. C. Alton Coulter, "Shipper/Receiver Issues in Transfers of Category I and II Quantities of Plutonium in Desirable Forms," Los Alamos National Laboratory report LA-10682-MS (to be published). 